\title{
Complex bend: Strong-focusing magnet for low-emittance synchrotrons
}

\author{
G. Wang, ${ }^{1}$ T. Shaftan, ${ }^{1, *}$ V. Smaluk, ${ }^{1}$ N. A. Mezentsev, ${ }^{2}$ S. Sharma, ${ }^{1}$ O. Chubar, ${ }^{1}$ \\ Y. Hidaka, ${ }^{1}$ and C. Spataro ${ }^{1}$ \\ ${ }^{1}$ National Synchrotron Light Source-II, Brookhaven National Laboratory, Upton, New York 11973 USA \\ ${ }^{2}$ Budker Institute of Nuclear Physics, Siberian Branch of Russian Academy of Sciences 11, Acad. \\ Lavrentieva Pr., Novosibirsk, 630090 Russian Federation
}

(Received 7 June 2018; published 25 October 2018)

\begin{abstract}
Modern synchrotron light sources are competing intensively to increase x-ray brightness and, eventually, approach the diffraction limit, which sets the final goal of lattice emittance. All recent synchrotron facility upgrades follow the multibend achromat approach by arranging small horizontal beta function and dispersion inside discrete bending dipoles. In this paper we propose a concept of a lattice element that we call "complex bend," which has the potential of becoming a main building block for low emittance lattices. The complex bend is a sequence of dipole poles interleaved with strong alternate focusing so as to maintain the beta function and dispersion oscillating at low values. Comprising the ring lattice with complex bends, instead of regular dipoles, will minimize the $H$-function and reduce horizontal emittance while localizing bending to a small fraction of the storage ring circumference, which should provide more space for insertion devices. In this paper we present the details of the complex bend, considerations regarding the choice of optimal parameters, and thoughts for its practical realization. We focus here on complex bend physics and engineering design, rather than integration of this complex bend into a specific ring lattice.
\end{abstract}

DOI: 10.1103/PhysRevAccelBeams.21.100703

\section{COMPLEX BEND: ELEMENT DESIGN AND MACHINE EMITTANCE}

The trend of minimizing the emittance of modern synchrotrons translates into reduction of dispersion and beta functions in their lattice dipoles. Most recent facility upgrades employ multibend achromat (MBA) lattices [1], i.e., introduce a number of dipoles with strong focusing quadrupoles interspersed, which helps maintain lattice functions at smaller values [2-5], compared with conventional (double-bend) DBA or (triple-bend) TBA lattice solutions. The number of dipoles per machine superperiod may vary between 5 (MAX-4) to 17 (MAX-4 upgrade) for the latest designs [6].

It is customary to relate ring emittance with the number of ring dipoles as

$$
\varepsilon_{x}=F \frac{E^{2}}{J_{x} N_{d}^{3}},
$$

where $F$ is a function of ring lattice, $E$ is the beam energy, $J_{x}$ is horizontal partition number and $N_{d}$ is the number of

\footnotetext{
*Corresponding author. shaftan@bnl.gov

Published by the American Physical Society under the terms of the Creative Commons Attribution 4.0 International license. Further distribution of this work must maintain attribution to the author(s) and the published article's title, journal citation, and DOI.
}

dipoles in the ring. In [7] we proposed to substantially decrease the beam emittance by increasing the number of dipoles and converting them into a single element with dipole poles of the same field polarity separated by strong quadrupoles with the alternating polarity. To illustrate our approach, we recall that advanced photon source (APS) contains $40 \times 2=80$ dipoles and APS-U is based on $40 \times 7=280$ dipoles. In the concept below we will be considering 1200 bends in a ring treated as separate poles of about 100 complex structures.

The concept of the complex bend is shown in Fig. 1. The element provides distributed focusing and bending of the particle beam (Fig. 1). A single cell is featured by the betatron phase advance, which depends on a combination of field gradients in quadrupole and field in the dipole magnets, their lengths and the distance between the consecutive poles.

Emittance in (1) depends on the integral of the $H$-function:

$$
H=\gamma_{x} \eta^{2}+2 \alpha_{x} \eta \eta^{\prime}+\beta_{x} \eta^{\prime 2}
$$

where $\left(\beta_{x}, \alpha_{x}, \gamma_{x}\right)$ are the horizontal Twiss functions and $\left(\eta, \eta^{\prime}\right)$ are the dispersion and its derivative. We approximated the beta function $\beta_{x}$ and dispersion $\eta$ for a single cell of complex bend with the following expression:

$$
\left[\begin{array}{c}
\beta_{x}(s) \\
\eta(s)
\end{array}\right] \approx\left[\begin{array}{c}
\bar{\beta}_{x} \\
\bar{\eta}
\end{array}\right]-\left[\begin{array}{c}
\Delta \beta_{x} \\
\Delta \eta
\end{array}\right] \cdot \cos \left(k_{C B} \mathrm{~s}\right)
$$



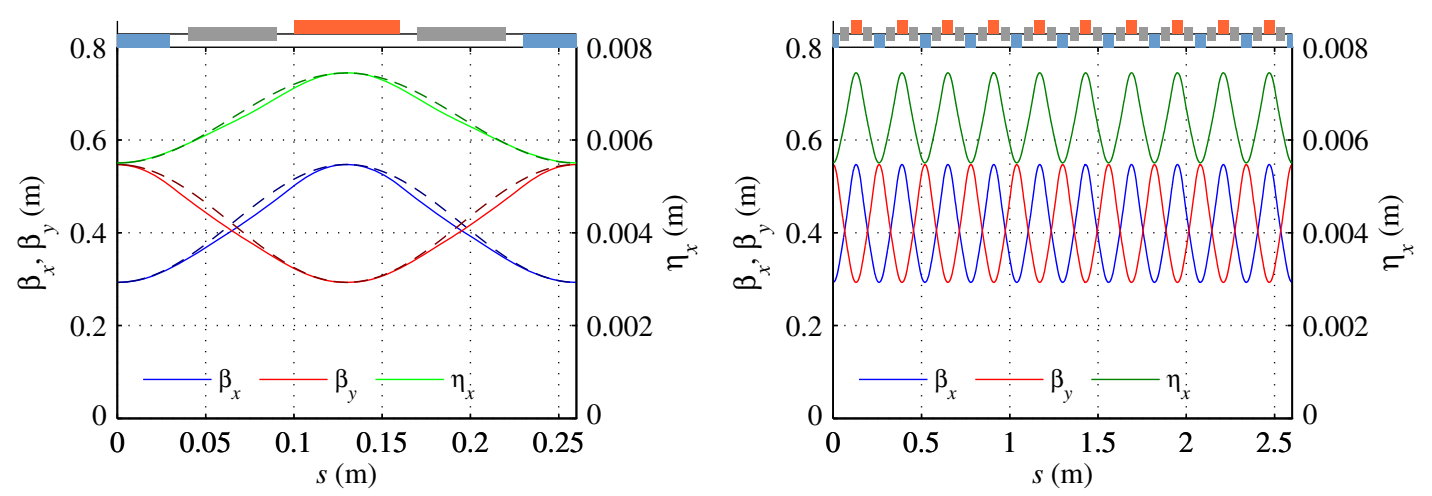

FIG. 1. The left figure shows a single cell of complex bend, and Twiss functions therein. The solid lines represent computer simulations; the dashed lines represent the approximate analytical solutions (3). The cell consists of two quadrupoles separated by short dipoles. The right figure shows an assembly of ten cells representing a complete complex bend element.

which is quite accurate for the relatively small phase advances that we consider in our cases of interest. Here $L_{C B}=$ $2\left(L_{Q}+L_{B}+2 L_{D}\right)$ is the length of the cell, $L_{Q}, L_{B}$, and $L_{D}$ are lengths of the complex bend quadrupole, dipole, and drift, respectively, and $k_{C B}=\frac{2 \pi}{L_{C B}}$. We used these expressions to find synchrotron radiation integrals for a given ring lattice and to obtain analytic dependencies of the $H$-function (2) on the dipole and quadrupole fields. We worked out analytic expressions of $\bar{\beta}_{x}, \Delta \beta_{x}$ and $\bar{\eta}, \Delta \eta$ for the case when $K_{1 F}=-K_{1 D}$ [7].

Using these expressions, we calculate emittance of the ring with the complex bends as

$$
\varepsilon_{x} \approx C_{q} \gamma^{2} \frac{\bar{\eta}^{2}}{R_{d} \bar{\beta}_{x}}
$$

where $C_{q}=3.832 \times 10^{-13} \mathrm{~m}$, and $R_{d}$ is the bending radius in the dipole poles. Throughout this paper we assume the ring energy as that of NSLS-II $(3 \mathrm{GeV})$.

Chromaticity introduced by a single complex bend magnet with $N_{p}$ cells can be estimated as

$$
\begin{aligned}
\xi_{x, y} \approx & -\frac{N_{p}}{4 \pi}\left[2\left(K_{1 F}-K_{1 D}\right) \frac{\Delta \beta_{x, y}}{k_{C B}} \sin \left(\frac{k_{C B} L_{Q}}{2}\right)\right. \\
& \left. \pm\left(K_{1 F}+K_{1 D}\right) L_{Q} \bar{\beta}_{x, y}\right]
\end{aligned}
$$

In the case of $K_{1 F}=-K_{1 D}=K_{1}$ this expression simplifies to

$$
\xi_{x, y} \approx-\frac{N_{p}}{\pi} K_{1} \frac{\Delta \beta}{k_{C B}} \sin \left(\frac{k_{C B} L_{Q}}{2}\right),
$$

where $\Delta \beta=\Delta \beta_{x}=\Delta \beta_{y}$. We note that the chromaticity for a Focusing lens-drift-Defocusing lens-drift (FODO) lattice in the thin lens approximation depends only on the tune shift per cell [8].

Now we will explore the range of parameters for the complex bend and discuss its scaling with field values and gradients. In the following discussion, we selected four betatron phase advances per cell of the complex bend $(0.06,0.08,0.105,0.14)$ in the unit of $2 \pi$ radians. We used transport matrices to compute periodic lattice solutions and extract lattice functions, emittance and chromaticity. We studied scaling of required $K_{1}$ with respect to the length of the magnet $L_{\mathrm{mag}}=N_{p} L_{C B}$, where $N_{p}=10$ is the number of cells per complex bend. Figure 2 shows the scaling of the magnet length and the ring emittance, with the focusing gradient $K_{1}$. The large dark dots represent the case shown in Fig. 1. The lines in the emittance plot represent the approximate analytical solutions calculated with Eq. (4) following [7], while the small colored dots represent the lattice simulation results.

We conclude that the highest technically achievable gradient limits the cell length and, in turn, the total length of the complex bend. Lower values of machine emittance require a large phase advance per cell and high values of $K_{1}$. As follows from Fig. 2, the emittance is a rapidly changing function of the quadrupole gradient. Therefore, future $R \& D$ on the complex bend magnet will be focused, as the first step, on demonstrating the highest achievable gradients on the scale of a single cell, then on integrating multiple cells into a single unit. The number of dipole poles and their field also define the ring emittance and are subject to optimization for a given ring lattice. The complex bend with $K_{1}=100 \mathrm{~m}^{-2}$ totals chromaticity of -1.1 per single element, which amounts to the chromaticity of -66 for the ring comprised of 60 dipoles with the total angle of $2 \pi$, delivering a ring emittance of $135 \mathrm{pm}$ rad. To minimize the strength of chromatic sextupoles, the optics between complex bends will need to be designed to amplify dispersion and beta functions.

It is worth pointing out an advantage of using complex bends in a ring lattice as compared with the MBA approach. Due to the compact size of the element, the dispersion between two complex bends (creating an achromat cell, as in the DBA lattice) can be amplified to a much higher value as compared with the tiny dispersion inside the 

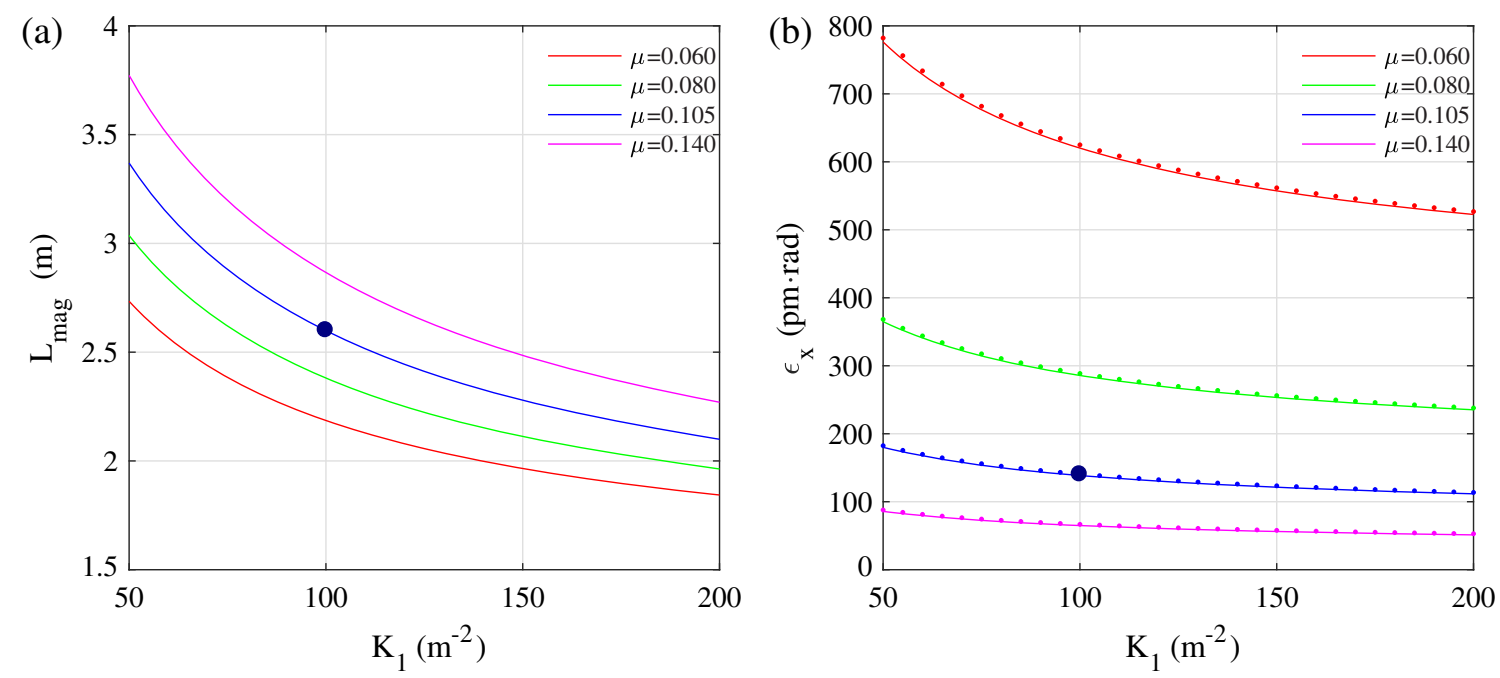

FIG. 2. Magnet length $L_{\text {mag }}$ (a) of a single magnet with ten cells and emittance $\varepsilon_{x}$ (b) of a ring composed of such magnets, as functions of the focusing gradient $K_{1}=K_{1 F}=-K_{1 D}$.

element itself. Progression of the MBA lattices towards low emittance meets an important limitation on the value of dispersion along the cell which, in turn, limits the total count of dipoles in the ring.

\section{COMPLEX BEND: COMPARISON WITH THE REGULAR DIPOLE IN A DBA LATTICE}

Due to mechanical constraints on the overall length of a single structure, the period of a complex bend must be short $(\sim 10 \mathrm{~cm})$; this results in high gradients of the pole fields. In Fig. 3 we present a comparison between an NSLS-II bending dipole and the complex bend corresponding to the same magnet bending angle $\left(6^{\circ}\right)$ and magnetic length. For this particular example we have chosen $K_{1 F}=$ $100 \mathrm{~m}^{-2}$ and $K_{1 D}=-80 \mathrm{~m}^{-2}$. This gives us additional horizontal focusing and helps to reduce horizontal beta function and dispersion, lowering the $H$-function by a factor of 2 as compared to $K_{1 D}=-K_{1 F}$, at the expense of a somewhat higher vertical beta function.

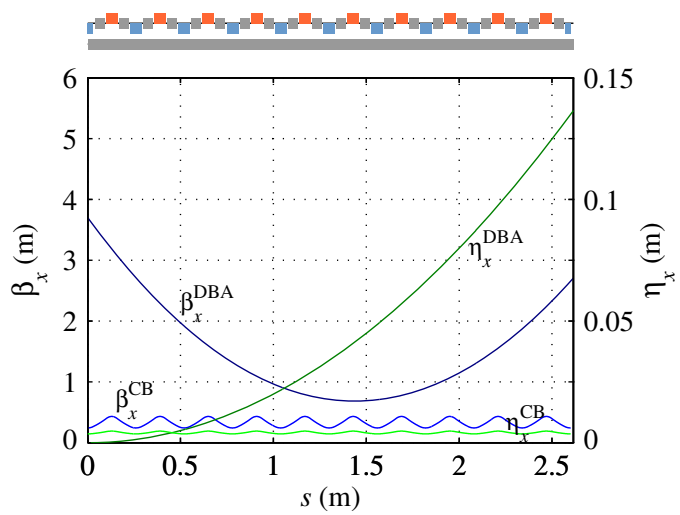

As follows from the plots, the complex bend results in smaller beta functions and dispersion, thus, leading to a smaller integral of $H$-function and emittance (Fig. 3, right). Table I lists parameters of the NSLS-II bending dipole against those for the complex bend model that we used in our calculations.

As follows from Fig. 2, the required range of gradients $K_{1}$ is at the level of $50 \mathrm{~m}^{-2}$. For a beam energy of $3 \mathrm{GeV}$ this translates into the requirement of a quadrupole gradient in the range of $500 \mathrm{~T} / \mathrm{m}$. A combination of $K_{1}$ and pole length values as shown above corresponds to the focusing distance of $20 \mathrm{~cm}$ for the quadrupole poles inside the complex bend element.

The advantage of a complex bend over a conventional dipole increases with the length of the complex bend, due to the natural divergence of lattice function along the bend (Fig. 3). The length of a complex bend is limited by the practical considerations of building a long, strong-focusing magnet. With a long complex bend, radiation emitted in

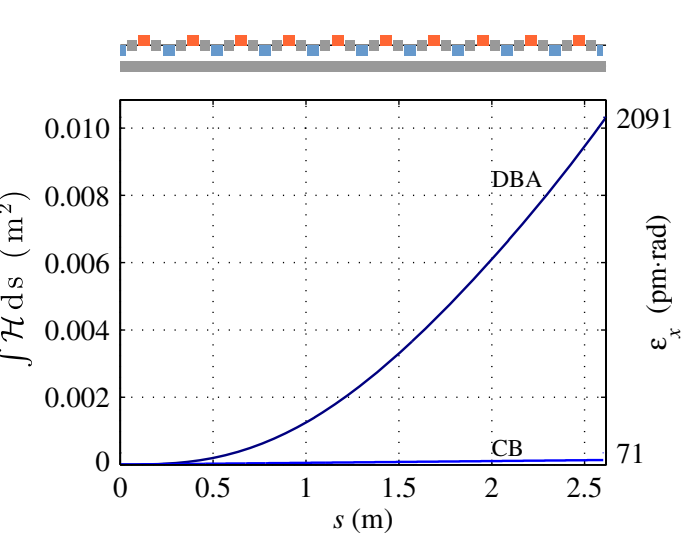

FIG. 3. Comparison between the NSLS-II bending dipole and the complex bend. The left plot shows the beta function and dispersion, and the right plot presents the integral of the $H$-function from 0 to $s$ for the two cases. The corresponding emittance values are shown on the right scale of the right plot $(2.1 \mathrm{~nm}$ rad is the natural NSLS-II lattice emittance). 
TABLE I. Parameters of the NSLS-II dipole and complex bend used in the calculation.

\begin{tabular}{lcc}
\hline \hline & NSLS-II dipole & Complex bend \\
\hline Length, m & 2.6 & 2.6 \\
Bending field, $\mathrm{T}$ & 0.4 & 1.05 \\
Cell length, $L_{C B}, \mathrm{~cm}$ & $\ldots$ & 26 \\
Bending angle, rad & 0.105 & 0.105 \\
$K_{1}, \mathrm{~m}^{-2}$ & 0 & $+100 /-80$ \\
$\beta x_{\max } / \beta x_{\min }, \mathrm{m}$ & $3.7 / 0.7$ & $0.42 / 0.24$ \\
$\eta_{\max } / \eta_{\min }, \mathrm{mm}$ & $137 / 0$ & $4.72 / 3.58$ \\
\hline \hline
\end{tabular}

the complex bend field will impinge on the inner walls of the aperture; thus, provisions need to be made to extract radiation from inside the element. We also note that aligning separate poles and making precise magnetic measurements become impractical for a long complex bend structure with many cells.

\section{COMPLEX BEND: DESIGN CONSIDERATIONS AND PRACTICAL LIMITATIONS}

Figure 4 illustrates the engineering concept behind the complex bend. The element consists of short quadrupoles of alternating polarity, separated by short dipoles. The element's axis is bent following the small bending angles from the dipole poles.

The distance between consecutive quadrupoles and dipoles should be sufficiently long to minimize areas with combined dipole and quadrupole fields, as well as to reduce the field multipoles. Overlapping dipole and quadrupole field components lead to a nonzero synchrotron integral $I_{4}$, which may result in the partition number $J_{z}$ becoming negative.

We should mention that yet another option of the complex bend is in arranging a set of only quadrupole poles (i.e., without dipole poles at all) and shifting every pole along the $\mathrm{x}$ direction by a certain amount to produce dipole field bending the beam orbit. These horizontal

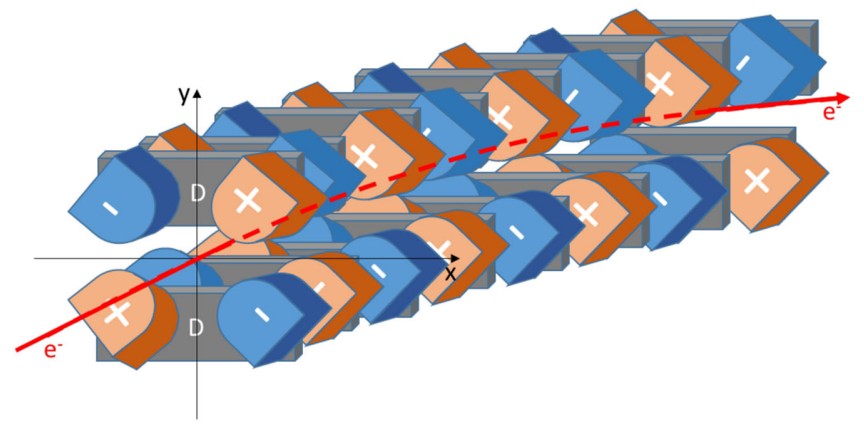

FIG. 4. Cartoon illustrating the concept of the complex bend.

translations of focusing and positive quadrupoles need to be carefully balanced to maintain the synchrotron integral $I_{4}$ close to zero.

In Table II we calculated parameters of one of the versions of the complex bend as it would have been installed on the NSLS-II storage ring.

The emittance of a ring lattice comprised of complex bends with the parameters described in Table II would be as low as $71 \mathrm{pm}$ rad; compare this with the value set by the existing NSLS-II DBA lattice of $2100 \mathrm{pm}$ rad.

Since the complex bend requires fields around $5 \mathrm{~T}$ (Table II), we expect to use superconducting magnets as the basis of its construction; however, permanent magnet solutions will be feasible at lower levels of gradients. In Fig. 5 we present a preliminary design of the superconducting quadrupole producing the field gradient in excess of $500 \mathrm{~T} / \mathrm{m}$ at $4.2 \mathrm{~K}$, at the current of $720 \mathrm{~A}$ in 210 turns per coil with wire diameter of $0.93 \mathrm{~mm}$ and a pole width of $10 \mathrm{~mm}$. The bore diameter is conservatively chosen at $20 \mathrm{~mm}$ (compare with the Table II value of $10 \mathrm{~mm}$ ).

The overlap in the drift between two consecutive poles generates field multipoles. After finding that the $H$-function is a sensitive function of field falloff at the magnet edges, we studied the optimal cell geometry to minimize the $H$-function. Our preliminary analysis of the magnet geometry, presented in Fig. 5, demonstrated the level of required gradients. Figure 6 shows the dependence

TABLE II. Set of sample parameters of the complex bend as hypothetically "installed" at NSLS-II, i.e., every DBA dipole replaced by the complex bend alternative.

\begin{tabular}{lclc}
\hline \hline Number of poles in the ring & 1200 & X-shift of orbit per dipole, mm & 145 \\
Angle per pole, mrad & 5.24 & $K_{1 F} / K_{1 D}, \mathrm{~m}^{-2}$ & $100 /-80$ \\
Number of cells in the ring & 600 & Magnetic gradient $d B / d x, \mathrm{~T} / \mathrm{m}$ & 1000 \\
Number of complex bends & 60 & Stay-clear aperture in quad, mm & 10 \\
Number of cells in complex bend & 10 & Field on stay-clear edge, T & 5.00 \\
Angle per element, mrad & 105 & Critical wavelength, A & 1.98 \\
Quad pole length, cm & 6 & Critical photon energy, keV & 6.27 \\
Dipole pole length $L_{D}, \mathrm{~cm}$ & 5 & SR opening half angle at $\lambda_{c}, \mathrm{~m}$ & $1.70 \times 10^{-4}$ \\
Drift between poles, cm & 1 & SR half spot size at $\lambda_{c}$ at dipole exit, m & $4.43 \times 10^{-4}$ \\
Cell length, cm & 26 & Energy loss per revolution, keV & 754.05 \\
Total element length, m & 2.6 & Circulating current, A & 0.50 \\
Magnetic field $B_{d}, \mathrm{~T}$ & 1.047 & Radiated power in the ring, kW & 377.03 \\
Bending radius $R_{d}, \mathrm{~m}$ & 9.55 & Radiated power per dipole, kW & 6.28 \\
\hline \hline
\end{tabular}




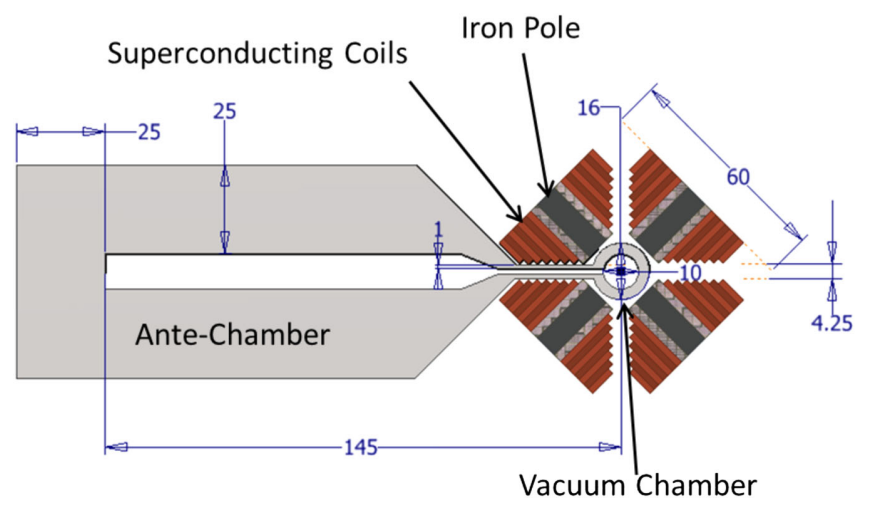

FIG. 5. Preliminary layout shows a cross section of the quadrupole pole and vacuum chamber for the extraction of synchrotron radiation generated by the poles.

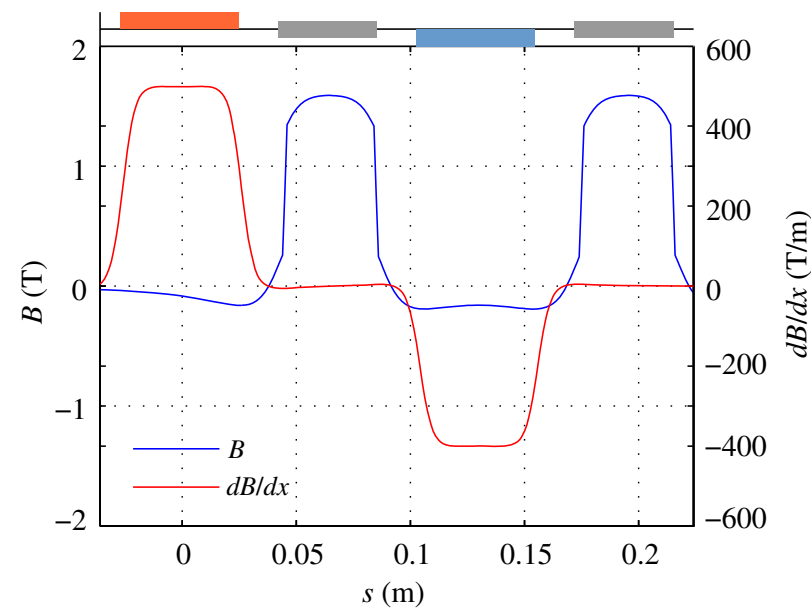

FIG. 6. Magnetic field calculations for a single cell of the complex bend.

of dipole and quadrupole field components as a function of distance along a single cell of the complex bend.

For the 2.6-m-long complex bend, the radiation fan crosses the footprint given by the bore with the radius of

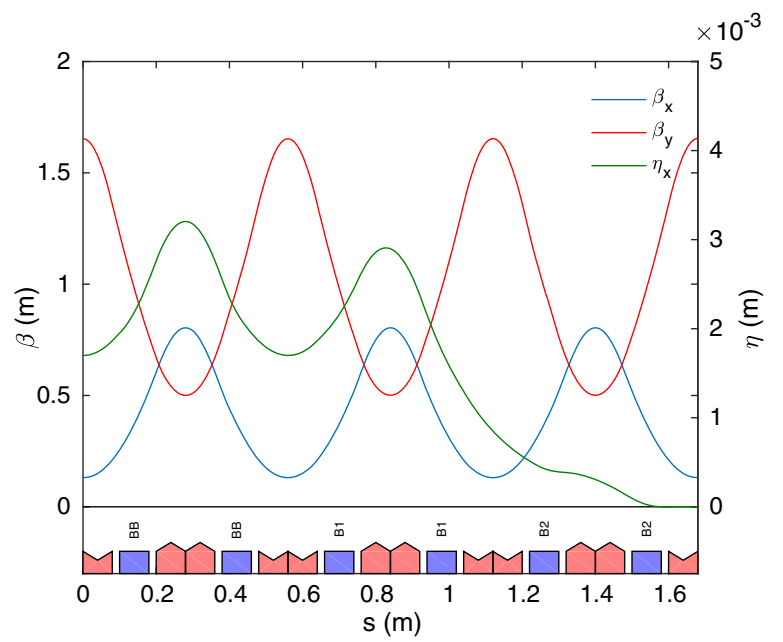

$1 \mathrm{~cm}$ over the length of the whole unit. The vacuum chamber geometry allows for opening space between the quadrupole poles so that the radiation will have an escape path (Fig. 5) towards the absorber, which will be located outside of the cryostat. This is one of the challenges that needs to be addressed in the engineering design of the complex bend.

We note that the dipole pole length is always longer than the formation length of synchrotron radiation for the case of practical interest. Indeed, at critical photon energy, $\frac{R_{d}}{\gamma} \approx 1.6 \mathrm{~mm} \ll L_{D}=50 \mathrm{~mm}$ for the energy of $3 \mathrm{GeV}$. On the other hand, radiation from the quadrupole poles is much less intense than that from the dipoles. While passing a quadrupole pole, the beam sees the field $B_{q} \approx d B / d x\left(\Delta x+\sqrt{\varepsilon_{x} \bar{\beta}_{x}+\delta^{2} \bar{\eta}^{2}}\right)$, where $\Delta x$ is orbit misalignment and $\delta$ is the energy spread in the beam. For misalignments of $50 \mu \mathrm{m}$ and horizontal beam size of $100 \mu \mathrm{m}$ we get $B_{q} \approx 0.06 \mathrm{~T}$, which is much smaller than the case of the dipole poles, $B_{d} \approx 1.05 \mathrm{~T}$.

Strict orbit alignment through the multipole centers in supposedly zero-bending regions is expected to be important in preserving the low emittance. Without such alignment, these multipoles act as additional bending elements and generate dispersion errors. This results in increased beam size, contributed both from increased emittance $\left(\sqrt{\varepsilon_{x} \overline{\beta_{x}}}\right)$ and from nonzero dispersion $(\delta \bar{\eta})$.

Another challenge is integration of the complex bend into a storage ring lattice. Managing the nonlinear ring lattice will require clean separation of (a) the dispersive regions of the machine with nonzero bending (localized to within the complex bends) and (b) dispersive regions with zero bending, where dispersion will need to be maximized and where the chromatic sextupoles will be located. To address this challenge, we are designing a dispersion suppressor and a dispersion amplifier-two elements that manage dispersion and Twiss functions at the entrance and exit of the complex bend.

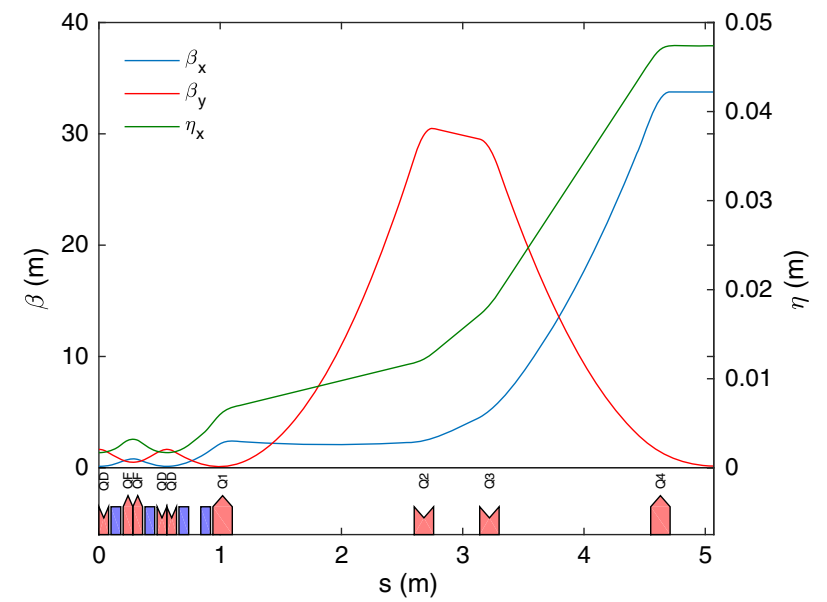

FIG. 7. Illustration of dispersion suppressor (left plot) and dispersion amplifier (right plot). 
An example of the dispersion matching modules is presented in Fig. 7. To assess the negative impact of these modules on the ring emittance, we considered a 30-period DBA-like lattice, consisting of two $2.2 \mathrm{~m}$ long complex bends per half of the lattice period. The overall length of an eight-cell element in this example is 2 modules $\times 2.2 \mathrm{~m}=4.4 \mathrm{~m}$, as compared with $2.6 \mathrm{~m}$ long complex bend discussed in Secs. I and II, which reduced the emittance from 71 to $16.8 \mathrm{pm}$ rad without taking into account the dispersion matching modules. We used moderate field gradients of 440 and $-300 \mathrm{~T} / \mathrm{m}$, which are consistent with our magnetic model discussed above.

The dispersion suppression is realized by adding a separate matching element at the end of the complex bend. The field strength of the dipoles in the element is optimized to reduce the values of dispersion and its derivative to be 0 . The resulting angle values are $\mathrm{B}_{1}=4.1 \mathrm{mrad}$ and $\mathrm{B}_{2}=$ $2.4 \mathrm{mrad}$ for the two poles of the dispersion suppressor, the sum of which is equivalent to the bending angle produced by a single dipole pole of a regular complex bend cell $(\mathrm{BB}=6.5 \mathrm{mrad})$. We calculated values of the synchrotron integrals contributing to the horizontal emittance, $I_{2}$ and $I_{5}$, shown in Table III, using the values of Twiss functions and dispersion presented in Fig. 7 (left plot).

The dispersion amplification is enabled by adding a separate dipole-quadrupole pair at the exit of the complex bend. The contribution to $I_{2}$ and $I_{5}$ from the dispersion amplifier (Fig. 7, right plot) is a small fraction of the values corresponding to the complex bend element (Table III). We note that Fig. 7 illustrates only one half of the dispersion bump required for fitting the chromatic sextupoles in the ring lattice.

After integration of the dispersion matching modules the ring emittance increases from 16.8 to $17.1 \mathrm{pm}$ rad ( $2 \%$ increase). We note that the dispersion amplifier, together with the sparse optics downstream, amplifies dispersion to the level of $50 \mathrm{~mm}$ while providing sufficient split of $\beta_{x}$ and $\beta_{y}$ in between the complex bends for the chromaticity correction. The spacing between Q1 and Q2, as well as between Q3 and Q4, leaves substantial room for chromatic sextupoles and other machine elements. Insertion devices could be potentially installed between Q1 and Q2, given the relatively small dispersion.

TABLE III. Synchrotron integrals and chromaticity corresponding to the complex bend, dispersion suppressor and dispersion amplifier.

\begin{tabular}{lcccc}
\hline \hline & $\begin{array}{c}I_{2} \times 10^{-3} \\
{\left[\mathrm{~m}^{-1}\right]}\end{array}$ & $\begin{array}{c}I_{5} \times 10^{-9} \\
{\left[\mathrm{~m}^{-1}\right]}\end{array}$ & $\xi_{x}$ & $\xi_{y}$ \\
\hline Complex bend cell & 1.07 & 1.36 & -0.35 & -0.28 \\
Dispersion suppressor & 0.57 & 0.31 & -0.70 & -0.56 \\
Dispersion amplifier & 1.07 & 1.98 & -0.42 & -0.53 \\
Complex bend element & 8.55 & 10.9 & -2.81 & -2.25 \\
$\quad(8$ cells $)$ & & & & \\
\hline \hline
\end{tabular}

\section{SUMMARY}

In this paper we compared the properties of two lattices based on different concepts (DBA and complex bend) and found that the latter can lead to substantial (factor of more than 30) emittance reduction within a certain range of the field properties. As the bending is localized to a relatively small portion of the ring circumference, the lattice based on complex bends may deliver substantial extra space for insertion devices in a synchrotron light source.

As a rule, every modern upgrade project replaces DBA or TBA lattices by going to 7- or 9-BA solutions and gaining a factor of 2 to 4 in the number of dipoles. The most aggressive path is chosen by the MAX-4 upgrade [6] that considers a 17-BA lattice, taking over most of the ring circumference by the bending arc elements. This paper describes a new concept: replacing a multitude of separate lattice elements by a set of multiple poles combined into the same magnet structure. This concept keeps only a few bending elements in the ring, but every bend consists of the 10 to 20 dipole poles, enabling a large reduction of machine emittance.

Indeed, for an energy of $3 \mathrm{GeV}$ and reasonable parameters of an average dispersion $\bar{\eta}$ of $1 \mathrm{~mm}$, a bending radius $R_{d}$ of $10 \mathrm{~m}$ and a beta function $\bar{\beta}_{x}$ of $10 \mathrm{~cm}$, we obtain via (4) an emittance at the level of $10 \mathrm{pm} \mathrm{rad,} \mathrm{which} \mathrm{implies}$ diffraction-limited performance at the photon energy of $10 \mathrm{keV}$. During our recent iteration on the complex bend lattice we found that the emittance of $17 \mathrm{pm}$ rad is feasible for a ring of the NSLS-II scale with 120 complex bends and gradients under $500 \mathrm{~T} / \mathrm{m}$.

We conclude this paper with the following few remarks. The FODO-like structure is natural for many types of circular machines, and high-field magnet lattices were implemented in fixed field alternating gradient (FFAG) lattices, in particular. An insightful example of a compact and completely superconducting ring lattice is PAMELA, a nonscaling FFAG developed in the UK [9]. The field in the main dipoles amounts to $5 \mathrm{~T}$. Our concept of complex bend uses a similar approach; however, FODO in the complex bend is arranged into a single periodic structure replacing a conventional bending magnet in order to minimize the $H$-function and, therefore, machine emittance.

On the other hand, the periodic sequence of FODO cells calls to mind a high-field wiggler. Our choice of a $5 \mathrm{~T}$ field producing gradients of $500 \mathrm{~T} / \mathrm{m}$ was deliberate. As we at NSLS-II are in the process of constructing a high-energy $\mathrm{x}$-ray beam line, we will be using a superconducting wiggler ( $\mathrm{SCW}$ ) with a $4.5 \mathrm{~T}$ peak dipole field on axis, a vertical aperture of $1 \mathrm{~cm}$, and a period length of $5 \mathrm{~cm} \mathrm{[10].}$ The technology for developing compact poles of SCWs with high field exists today and has reached a high degree of maturity [11].

Superbends (isolated poles with high magnetic field to extend the photon spectrum for user experiments) are easy to realize in complex bend structure: without increasing the 
device cost one could increase the field of several poles to $5 \mathrm{~T}$, at the penalty of somewhat increased emittance.

The complex bend is based on superconducting magnet technology and thus is far more complex than regular electromagnets. However, it will greatly reduce the complexity of power supplies and vacuum chambers, compared to machine design based on the MBA lattice, and will lead to very substantial space savings in the ring. Also, in modern light sources, superconducting magnet and rf technology has become standard: e.g., superconducting undulators, superbends and wigglers, where the fields can exceed $7 \mathrm{~T}$ at $4 \mathrm{~K}$, and, finally, the LCLS-II Free Electron Laser [12] that is based on a hundred superconducting rf structures operating at $2 \mathrm{~K}$. Our concept combines two recent advances in the area of accelerator engineering: miniaturization of magnetic elements (pioneered by MAX4 magnet design) and superconducting technology (e.g., RHIC, PAMELA, etc.).

\section{ACKNOWLEDGMENTS}

T. Shaftan expresses thanks to B. Parker for helpful discussions on the magnetic design of the complex bend cell. This research used resources of the National Synchrotron Light Source II, a U.S. Department of Energy (DOE) Office of Science User Facility operated for the DOE Office of Science by Brookhaven National Laboratory under Contract No. DE-SC0012704.

[1] D. Einfeld, J. Schaper, and M. Plesko, A lattice design to reach the theoretical minimum emittance for a storage ring, http://citeseerx.ist.psu.edu/viewdoc/download?doi=10.1.1 $.495 .2446 \&$ rep $=$ rep1\&type $=$ pdf.

[2] J-L. Revol et al., ESRF, Grenoble, France Proceedings of IPAC2014, Dresden, Germany (JACoW, Geneva,
Switzerland, 2014), pp. 209-212, MOPRO055, http:// inspirehep.net/record/1314242/files/MOPRO055.pdf.

[3] S. Henderson, Status of the APS upgrade project, in Proceedings of IPAC-2015, Richmond, VA, USA (JACoW, Geneva, Switzerland, 2015), pp. 1791-1793, TUPJE067, http://accelconf.web.cern.ch/AccelConf/IPAC2015/papers/ tupje067.pdf.

[4] C. Steier, J. Byrd, H. Nishimura, D. Robin, and S. De Santis, Physics design progress towards a diffraction limited upgrade of the ALS, in Proceedings of IPAC2016, Busan, Korea (JACoW, Geneva, Switzerland, 2016), pp. 2956-2958, WWPOW049, http://accelconf.web.cern .ch/AccelConf/ipac2016/papers/wepow049.pdf.

[5] L. Liu, X. R. Resende and A. R. D. Rodrigues, Sirius (BR): A new Brazilian synchrotron light source, in Proceedings of IPAC-2016, Busan, Korea (JACoW, Geneva, Switzerland, 2010), pp. 2811-2814, WEPOW001, https://accelconf.web .cern.ch/accelconf/IPAC10/papers/wepea006.pdf.

[6] P. F. Tavares, J. Bengtsson, and Á. Andersson, Future development plans for the MAX IV light source: Pushing further towards higher brightness and coherence, J. Electron Spectrosc. Relat. Phenom. 224, 8 (2018).

[7] T. Shaftan, V. Smaluk, and G. Wang, Concept of the complex bend, NSLS-II Technical Note No. 276, 2018.

[8] A. W. Chao and M. Tigner, Handbook of Accelerator Physics and Engineering, 3rd ed. (World Scientific, Singapore, 2006), p. 76.

[9] K. Peach et al., PAMELA overview, and status, in Proceedings of the International Particle Accelerator Conference, Kyoto, Japan (JACoW, Geneva, Switzerland, 2010), MOPEA021, pp. 112-114, http://accelconf.web .cern.ch/AccelConf/IPAC10/papers/mopea021.pdf .

[10] RSI for the superconducting wiggler source and front-end for the HEX beam line, NSLS-II record, Report No. PS-CXFD-RSI-HEX-001, 2017.

[11] N. Mezentsev and E. Wallen, Superconducting wigglers, Synchrotron Radiat. News 24, 3 (2011).

[12] J. Galayda, The LCLS-II: A high power upgrade to the LCLS, in Proceedings of IPAC-2018, Vancouver, BC, Canada (JACoW, Geneva, Switzerland, 2018), MOYGB2. 